\title{
IDENTIFICATION OF ISOMORPHISM AND DETECTION OF DISTINCT MECHANISM OF KINEMATIC CHAINS USING INVARIENT LABELING OF LINKS
}

\author{
S.R.Madan \\ Professor, Mechanical Engineering Department, Mahakal Institute of Technology and Management, UJJAIN, \\ (M.P), INDIA
}

\begin{abstract}
The problem of isomorphism among kinematic chains and of these derived mechanisms has been a hot area of research being done from last several years. The researchers so far have proposed many methods which are mainly based on characteristic polynomial and on some code based method to test the isomorphism among kinematic chains. In the present communication a new structural invariant based on link-link path distance (shortest) has been developed for a kinematic chain. Each link of kinematic chain is assigned as a unique label using the invariant. The set of link labels are combined in the form a numerical label of kinematic chain, called kinematic chain label $(K C L) . K C L$ is proposed as unique identifier for detection of isomorphism among kinematic chains, and also used to find distinct mechanism of a kinematic chain. Method is tested on several cases of single degree of freedom plannar kinematic chain successfully with simple joints.
\end{abstract}

Keywords: - Isomorphism, Kinematic chains, Mechanism, Invarient labeling, Distinct mechanism, path distance matrix.

\section{INTRODUCTION}

Structural analysis and synthesis of kinematic chains is tried to be carried out as an important aspect of mechanism design. In the early stage of mechanism design, it is helpful to determine all the distinct kinematic chains with the required number of links and degree of freedom. One very important problem encountered during structural synthesis of chains and the detection of possible isomorphism among the given chains. Several attempt ${ }^{(1-18)}$ were made to develop reliable and computationally efficient algorithms for automated test of isomorphism but each has its own shortcomings. Duplicate or isomorphic chain leads to redundant computation. In order to achieve greater efficient in computer aided mechanism design research for simple, reliable and effective methods is of great significance.

Several methods were proposed by researchers to test isomorphism among kinematic chains. Characteristic polynomial of adjacency matrix was proposed by Uicker and Raicu $^{(1)}$ for the time as a numerical measure of kinematic chain structure. Later it was found during the synthesis of kinematic chains by Mruthyunjaya ${ }^{(2)}$ that it was only a necessary condition and nonisomorphic chains having identical characteristic polynomial were detected. Characteristic polynomial of vertex - vertex degree matrix was proposed in (3) by Mruthunjaya and Balasubramanian. Discovery of counter examples led to the development of new indices characteristic polynomial of distance matrix was proposed by Dubey and Rao ${ }^{(4)}$. Caninical formation of upper triangular adjacency matrix of kinematic chain by relabeling of links was used to generate Max and Min code by Ambekar and Agrawal ${ }^{(5,6)}$.

An altogether different approach was made by Rao and coworkers and several indices based on Hamming number was proposed by them ${ }^{(7-10)}$.

Link's adjacent table (ACT) and complete adjacent table (CACT) obtained by successive replacement of link labels wise in a systematic manner so as to produce a unique string was proposed as a method to detect isomorphism by Chu and $\mathrm{CaO}^{(11)}$.

Ding and Huang proposed methods based on Canonical perimeter topological graph $^{(12)}$ and characteristic adjacency matrix $^{(13)}$.

Approaches based on artificial neural network, genetic algorithm and novel evolutionary approaches were also proposed by some researchers ${ }^{(14-16)}$.

Most of the methods reported so far require comlex algorithms to carry out the test. A broad review of the methods published was given Mruthyunjaya in ${ }^{(17)}$ and it was concluded that quest for computastionally efficient, reliable and simple methods is still on. Bearing the above in mind, a methodology based on link-link distances and degree of links of kinematic chain, 
invariant link labels and entropy of chains are proposed to detect isomorphism, best chain of plannar kinematic chains with their distinct mechanisms.

\section{ARCHITECT OF THE PROPOSED METHOD}

\subsection{Degree of link $d\left(\mathbf{l}_{i}\right)$}

The degree of a link actually represents the type of the link, such as binary, ternary, quaternary links etc. Let the degree of $\mathrm{i}^{\text {th }}$ link in a kinematic chain be designated $\mathrm{d}\left(\mathrm{L}_{\mathrm{i}}\right)$ and $\mathrm{d}\left(\mathrm{L}_{\mathrm{i}}\right)=2$, for binary link, $d\left(L_{i}\right)=3$, for ternary link, $d\left(L_{i}\right)=4$, for quaternary link and $\mathrm{d}\left(\mathrm{l}_{\mathrm{i}}\right)=\mathrm{n}$, for $\mathrm{n}$-nary link.

\subsection{Labeling of link $\left(\mathrm{V}_{\mathrm{i}}\right)$}

Usually, the canonical labels depend only on the connectivity of the links being labeled together with its immediate neighbours. However, in a closed kinematic chain, links are connected by joints so as to form loops and every link has a distinct relation with every other link in the form of distance between them which is constant and presented here by a matrix is called link path matrix of the chain. Bearing this in mind the usual canonical labeling is extended to include all links of the chain. Canonical label $\mathbf{V}$ of a link of a kinematic chain, defined here, is a sum of path weighted connectivity of the links. Each link $\mathbf{L}$ is assigned a label $\mathbf{V}_{\mathbf{i}}$ as follows -

$$
\mathrm{V}_{\mathrm{i}}=\sum_{\mathrm{J}=1}^{\mathrm{N}} 1 / 2^{\mathrm{Dij}} \cdot \mathrm{W}_{\mathrm{j}}
$$

Where $\mathrm{W}_{\mathrm{j}}$ is the weight of degree of jth link with related to total degree of kinematic chain. And $\mathrm{Wj}$ is related weight of degree of the links which is defined as the ratio between of $j^{\text {th }}$ link and total degree of kinematic chain given as

$$
\mathbf{W}_{\mathrm{j}}=\mathbf{d}\left(\mathbf{L}_{\mathrm{i}}\right) / \mathbf{d}(\mathbf{K C})
$$

To include all links but give a higher path weight to those closest to the link $\mathrm{L}_{\mathrm{i}}$ being labeled, a factor of $\left(1 / 2^{\mathrm{Dij}}\right)$ is included where, $\mathrm{D}_{\mathrm{ij}}$ is the distance between the links $\mathrm{L}_{\mathrm{i}}$ and $\mathrm{L}_{\mathrm{j}}$. This distance is defined as the shortest path between any two links of a chain. $\mathrm{N}$ is the total number of links in the chain being labeled.

Consider for example, six - link; single degree of freedom Watt and Stephenson chain shown in Figs., 1(a) and 1(b), respectively. Invariant link labels are calculated as-

For Watt chain shown in Fig. 1(a), relative weight of 6 links using eq. 2 are

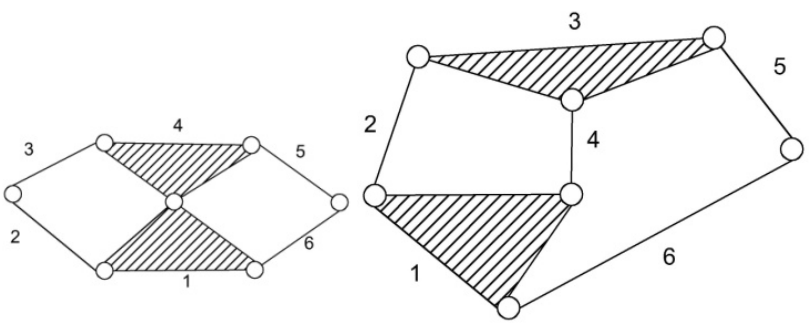

Fig. 1(a) Watt chain

Fig. 1(b) Stephenson chain

2

3

4 6

\begin{tabular}{|l|l|l|l|l|l|}
\hline 0.21428 & 0.14285 & 0.14285 & 0.21428 & 0.14285 & 0.14285 \\
& & & & & \\
\hline
\end{tabular}

And link-link path matrix is

\begin{tabular}{|l|l|l|l|l|l|l|}
\hline Link & $\mathbf{1}$ & $\mathbf{2}$ & $\mathbf{3}$ & $\mathbf{4}$ & $\mathbf{5}$ & $\mathbf{6}$ \\
\hline $\mathbf{1}$ & 0 & 1 & 2 & 1 & 2 & 1 \\
\hline $\mathbf{2}$ & 1 & 0 & 1 & 2 & 3 & 2 \\
\hline $\mathbf{3}$ & 2 & 1 & 0 & 1 & 2 & 3 \\
\hline $\mathbf{4}$ & 1 & 2 & 1 & 0 & 1 & 2 \\
\hline $\mathbf{5}$ & 2 & 3 & 2 & 1 & 0 & 1 \\
\hline $\mathbf{6}$ & 1 & 2 & 3 & 2 & 1 & 0 \\
\hline
\end{tabular}


Invarient label for any link is sum of path weight connectivity of all the links. Thus the

$$
v_{1}=\sum_{J=1}^{N} 1 /(2)^{D_{1 j}} \cdot W_{j}
$$

Thus invariant label $\mathbf{V}_{\mathbf{1}}$ for link 1 of Fig. 1(a)

$=1 / 2^{0} \bullet 0.21428+1 / 2^{1} \bullet 0.14285+1 / 2^{2} \bullet 0.14285+1 / 2^{1} \bullet$

$0.21428+1 / 2^{2} \bullet 0.14285+1 / 2^{1} \bullet 0.14285$.

$=0.5356$

Similarly for all the other links, labels can be calculated as shown below.

$\mathbf{V}_{\mathbf{2}}=0.4285, \mathbf{V}_{\mathbf{3}}=0.4285, \mathbf{V}_{\mathbf{4}}=0.5356, \mathbf{V}_{\mathbf{5}}=0.4285, \mathbf{V}_{\mathbf{6}}=$ 0.4285

So for Watt chain the labels are

[ $0.5356,0.4285,0.4285,0.5356,0.4285,0.4285$ ]

And Squared sum of links labels, defined as kinematic chain label(KCL)

$$
\mathrm{KCL}=\sum_{\mathbf{i}=1}^{\mathrm{N}} \mathrm{V}_{\mathbf{i}}
$$

and $\mathrm{KCL}_{\text {watt }}$ is 1.3085

Example 2 - Consider the two chains shown in Fig.2.

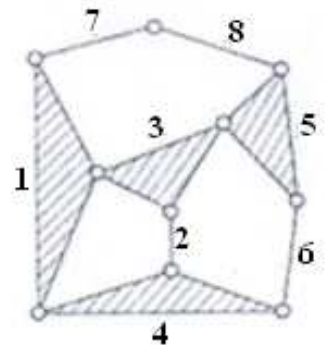

(a)
For Stephenson chain shown in Fig.1(b), the labels are $[0.5178,0.4642,0.5178,0.4642,0.4464,0.4464]$ and KCL is 1.36564

\subsection{DETECTION OF ISOMORPHISM}

The set of link labels can be directly be used to distinguish kinematic chains. To make it more meaningful the labels calculated above can be combined to generate a numerical code for a kinematic chain. Squared sum of links, defined as kinematic chain label (KCL) is proposed as an index for testing isomorphism. Two chains having identical KCL will be isomorphic to each other.

\section{Application of the Concept is Illustrated with the help of Several Examples.}

Example 1. Watt and Stephenson chain are shown in Fig. 1 (a) and in Fig. 1(b) respectively. Link labels calculated using equation (1), and (2) for Watt chain is [ 0.5356, 0.4285, $0.4285,0.5356,0.4285,0.4285]$ and KCL calculated by equation (3) is equal to 1.3085 and by the comparison of labels of links of kinematic chain (Watt chain) it is found that there two distinct mechanism will be obtained i.e for links $(1,4)$ and for links $(2,3,5,6)$.

For Stephenson chain the labels are

$[0.5178,0.4642,0.5178,0.4642,0.4464,0.4464]$ and $\mathrm{KCL}$ is 1.365964 .

By the comparison of KCL of Watt chain and Stephenson chain it is clear from the result that two chains are distinct.

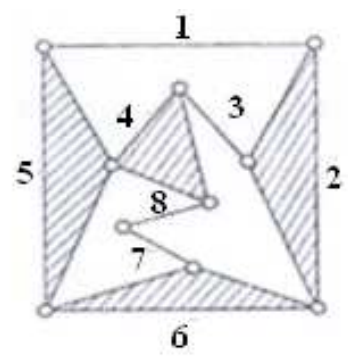

(b)

Fig. 2 Eight link isomorphic chains 
For Fig.2(a), the labels are

$[0.4625,0.375,0.4625,0.4375,0.450,0.3875,0.3625,0.3562]$ and $\mathrm{KCL}$ is 1.370820

For Fig.2(b), the labels are

$[0.375,0.4375,0.3875,0.45,0.4625,0.4625,0.3625,0.3562]$ and KCL is 1.370820

The labels and corresponding chain labels for both the figures are identical hence the chains are isomorphic.

Example 3- Consider the two chains shown in Fig.3.
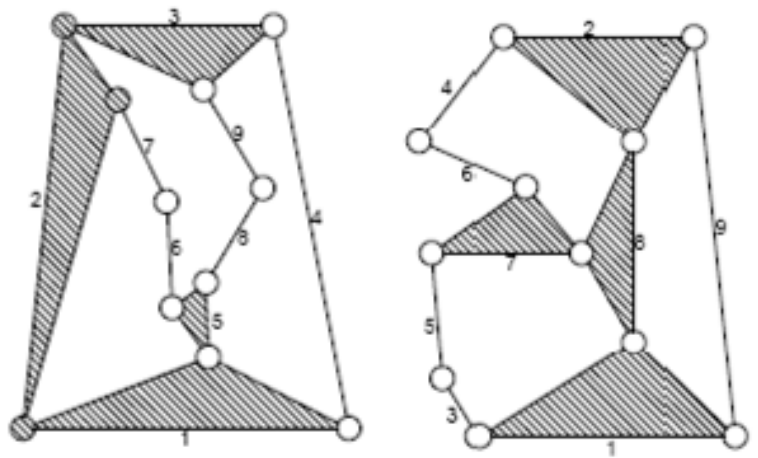

Figure 3(a)

\section{Nine links two degree of freedom kinematic chains}

For Fig.3(a), the labels and KCL are

$[0.4317,0.4317,0.4033,0.3522,0.4147,0.3352,0.3408$, $0.3352,0.3352]$ and $\mathrm{KCL}=1.2850$

For Fig.3(b), the labels and KCL are

$[0.40907,0.40607,0.3295,0.3295,0.3352,0.3352,0.4204$, $0.4545,0.3465]$ and KCL is 1.20004

The chain label and KCL for Nine link two degree of freedom chain shown in Fig. 3(a) and Fig. 3(b) are non identical, so it is clear from result that two chains are non-isomorphic.

Example 3- Three 12-bars non-isomorphic kinematic chains cited from ${ }^{(12)}$ are shown in Figs.3(a), 3(b) and 3(c) respectively. They have identical characteristic polynomial. The link labels and corresponding chain labels for the figures are:

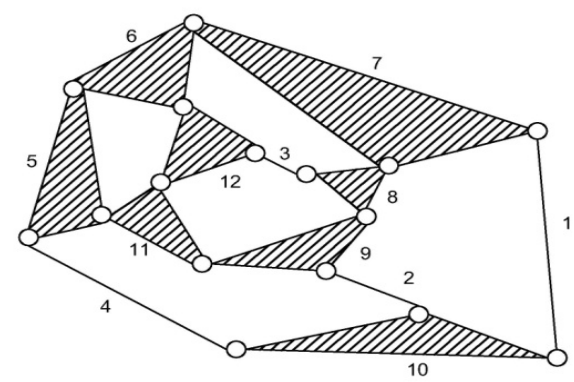

(a)

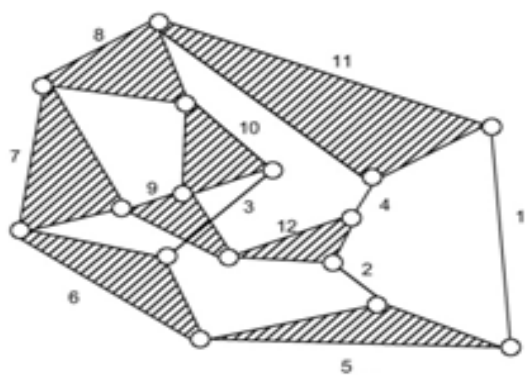

(b)

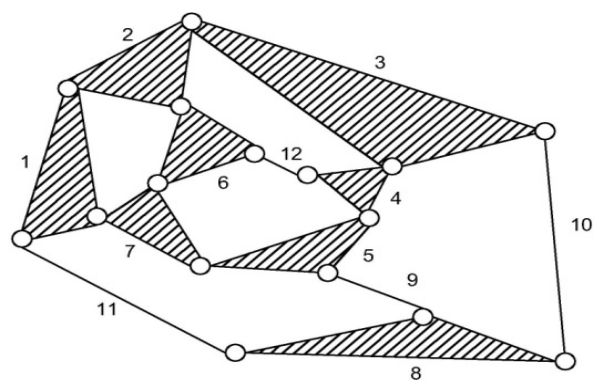

(c)

Fig 4 Twelve-link 1-dof kinematic chains 
Fig.4(a)

Link Labels: [ $0.2832,0.2832,0.2871,0.2792,0.3476$, $0.3554,0.3554,0.3476,0.3554,0.3027,0.3554,0.3417]$.

$\mathrm{KCL}=1.2764$

Fig. 4( c)

Link Labels : [ $0.3476,0.3554,0.3554,0.3476,0.3554$, $0.3417,0.3554,0.3027,0.2832,0.2832,0.2792,0.2871]$.

$\mathrm{KCL}=1.2764$

Fig. 4(b)

Link Labels : [ $0.2832,0.2832,0.2929,0.2617,0.3261$, $0.3359,0.3671,0.3554,0.3027,0.33007,0.3359,0.3359]$.
$\mathrm{KCL}=1.22147$

Chain shown in Fig. 4(a) and 4(b) are isomorphic to each other, only links are relabeled in a different manner. Result clearly show that the invariant is independent of relabeling of links. KCL for Fig 4 is different hence it clearly reflects that the chains are uniquely identified by KCL.

Example 4 , Complete set of 8 links, 1-degree of freedom kinematic chains is shown in Fig.5. The kinematic chain is calculated for each chain and is shown in Table 1. All of these chains have distinct value of KCL. The notation for each family title is given; ( number of ) quaternaries, ternaries and binaries. Thus 206 stand for two quaternaries, zero ternaries and six binaries.

Table 1 - Kinematic chain labels and DM of Six and Eight - link single degree - of - freedom kinematic chains.

\section{K.Chain Chain Family KCL Distinc Mechanism \\ No.}

\section{Six link single degree of freedom kinematic chain}

\begin{tabular}{|c|c|c|}
\hline Watt chain & 1.3085 & $(1,4),(2,3,5,6)=2$ \\
\hline Stephenson chain 024 & 1.365964 & $(1,3),(2,4),(5,6)=3$ \\
\hline
\end{tabular}

Eight link single degree of freedom kinematic chains

$\begin{array}{lll}\text { 1. } & 044 & 1.265625 \\ \text { 2. } & 044 & 1.35875 \\ \text { 3. } & 044 & 1.37082 \\ \text { 4. } & 044 & 1.314844 \\ \text { 5. } & 044 & 1.312031 \\ \text { 6. } & 125 & 1.338750 \\ \text { 7. } & 125 & 1.371016 \\ 8 . & 125 & 1.379727 \\ \text { 9. } & 044 & 1.328203 \\ \text { 10. } & 044 & 1.410625 \\ 11 . & 044 & 1.218672 \\ 12 . & 044 & 1.255781 \\ \text { 13. } & 125 & 1.410820 \\ 14 . & 125 & 1.330313 \\ 15 . & 206 & 1.3950 \\ 16 . & 206 & 1.49875\end{array}$

$(2,3,6,7),(1,4,5,8)=2$

$(1,2,5,6),(3,4,7,8)=2$

$1,2,3,(4,50,6,7,8=7$

$(1,5),(2,4), 3,(6,7), 8=5$

$(1,4)(2,8),(3,7),(5,6)=4$

$1,(2,6,7,8),(3,5), 4=4$

$1,2,3,4,5,6,(7,8)=7$

$1,2,3,4,5,6,7,8=8$

$(1,8),(2,7),(3,6),(4,5)=4$

$(1,3,4,7),(2,5,6,8)=2$

$1,2,3,4,5,(6,7), 8=7$

$(1,2,4,5),(3,6,7,8)=2$

$1,(2,8), 3,4,5,6,7,8=7$

$1,(2,5,8),(3,7),(4,6)=4$

$(1,4),(2,3,5,6,7,8)=2$

$(1,4),(2,5,6), 3,(7,8)=4$

Total D.M = 71 

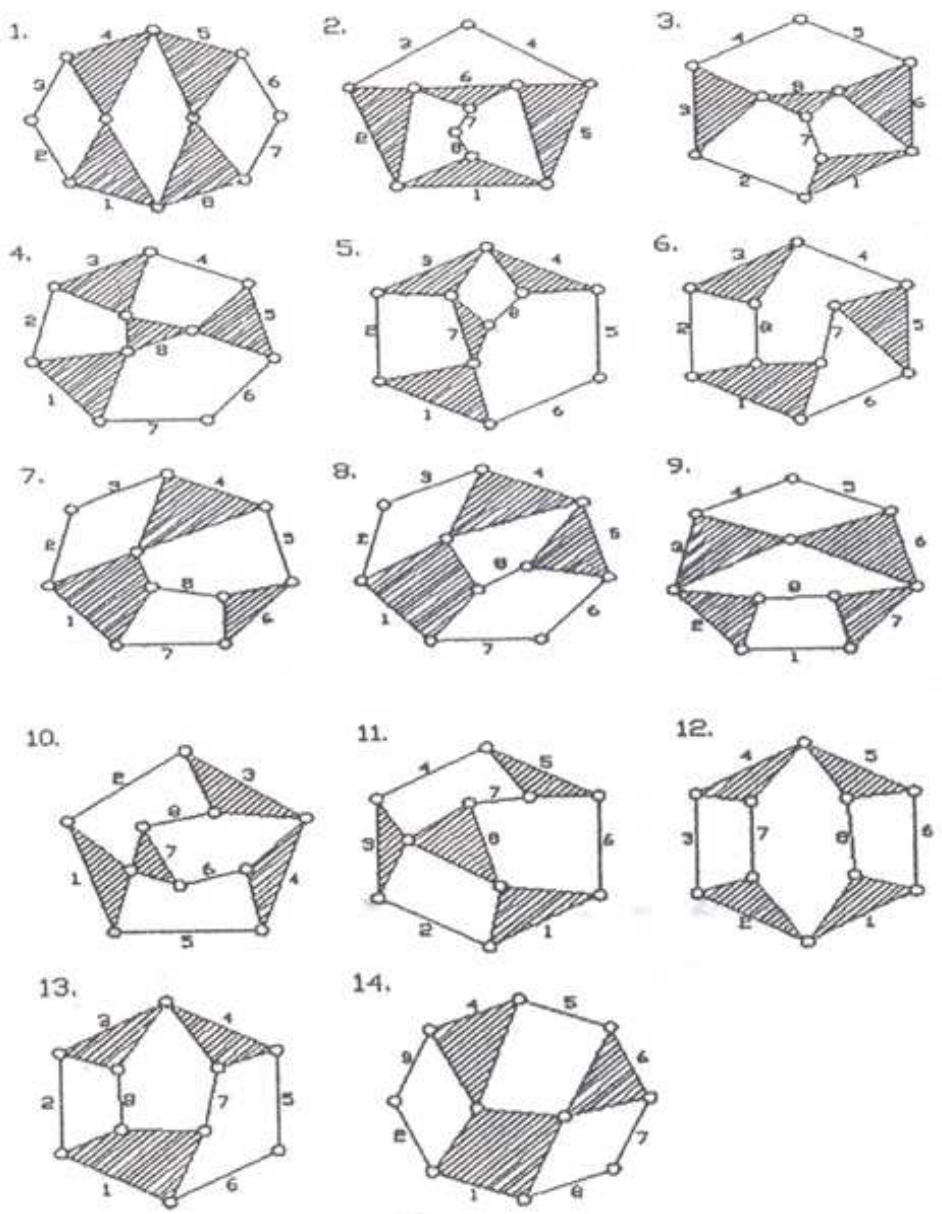

14.
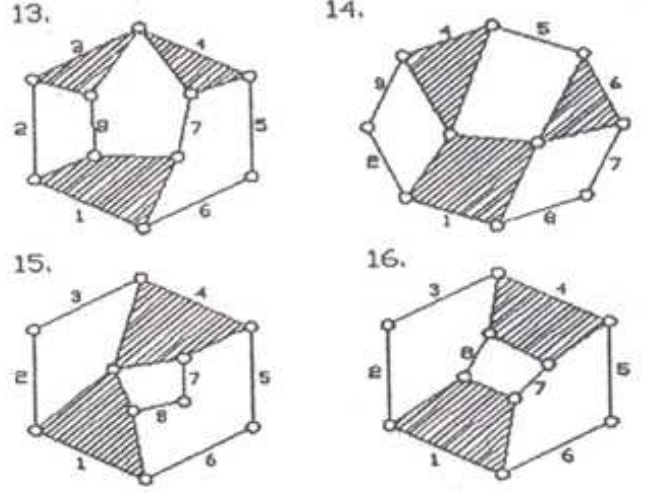

16.

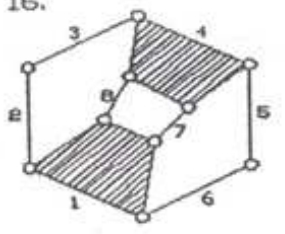

\section{REFERENCES}

\section{RESULT AND CONCLUSIONS}

In this paper a heuristic method for detection of isomorphism among kinematic chains is presented. The proposed method is also tested to obtain all DM derived from a family of kinematic chain. Method is simple, reliable and can easily be implemented on computer. A program is written in $\mathrm{C}^{++}$and entire calculations was carried out in a personal computer with Pentium duel core ES200 @ $2.5 \mathrm{GH}_{2}$ with 1 GB random access memory. With the help of present method kinematic chains can be checked with single numerical invariant. Also DM of a kinematic chain are derived from the family of 1 DOF 6 and 8 links in accordance with the result given by Vijayanath ${ }^{(18)}$ and Yadav $^{(19)}$ and is shown by table no-1.

[1] J.J.Uicker and Raicu, "A Method for the Identification of Recognition of Equivalence of Kinematic Chains" Mechanism and Machine Theory, vol.10, pp. 375-383, 1975.

[2] T.S.Mruthyunjaya, "A Computerized Methodology for Structural Synthesis of Kinematic Chains - Part I, II, III," Mechanism and Machine Theory, vol. 14, pp. 487-530,1984.

[3] T.S.Mruthyunjaya and H.R.Balasubramanian, "In Quest of reliable and Efficient Computational Test for Detection of Isomorphism in Kinematic Chains," Mechanism and Machine Theory, vol. 22, pp. 131-139, 1987. 
[4] R.K.Dubey and A.C.Rao, "New Characteristic polynomial - A Reliable Index to Detect Isomorphism between Kinematic Chains," Journal Indian Institute of Sciences, vol. 66, pp. 227-233,1986.

[5] A.G.Ambekar and V.P.Agrawal, "On Canonical Numbering of Kinematic Chains and Isomorphism Problem:Max Code," ASME paper no. 86-DET-169.

[6] A.G.Ambekar and V.P.Agrawal, "Canonical Numbering of Kinematic Chains and Isomorphism Problem:Min Code," Mechanism and Machine Theory, no. 22,pp. 463-461,1987.

[7] A.C.Rao and D.V.Raju, "Application of the Hamming Number Technique to Detect Isomorphism among Kinematic Chains and Tnversions," Mechanism and Machine Theory, no. 26, pp. 55-75, 1991.

[8] A.C.Rao and C.N.Rao "Loop Based Pseudo Hamming Values-1.Testing Isomophism and Rating Kinematic Chains," Mechanism and Machine Theory, 1993.

[9] A.C.Rao and A.Jagadeesh, "Isomorphism, Inversions, Symmetry and Parallelism with Particular Reference to Manipulators" IE(I) Journal-MC, vol.80,pp.7073,1999.

[10] A.C.Rao and J. Anne, "Topology Based Characteristics of Kinematic Chains; Workspace Rigidity, Input-Joint and Isomorphism," Mechanism and Machine Theory, no. 33, pp. 625-638, 1988.

[11] J.K.Chu and W.-Q. Cao," Identification of Isomorphism among Kinematic Chains and Inversions using Link,s Adjacent-Chain-Table," Mechanism and Machine Theory, vol. 29, no.1, pp. 53-58,1994.

[12] H.Ding and Z.Huang, "The Establishment of the Canonical Perimeter Topological Graph of Kinematic Chains and Isomorphism Identification," ASME Journal of Mechanical Design,p.915, 2007.

[13] H.Ding and Z.Huang, "Isomorphism Identification of Graph; Especially for Graph of Kinematic Chains," Mechanism and Machine Theory, vol. 44, pp. 122-139, 2009.

[14] G.G.Marin, D.L. Rodriguez and E.M Casermeiro, "A New Multivalued Neural Network for Isomorphism Identification of Kinematic Chains," ASME Journal of COMPUTING and Information Science in Engineering, 2010.

[15] A.C.Rao, "A Genetic Algorithm for Topological Characteristics of Kinematic chains," ASME Journal of Mechanical Design,p.228, 2000.

[16] R.Xiao, Z.Tao and Y. Liu, "Isomorphism Identificationof Kinematic Chains Using Novel Evolutionary Approaches," ASME Journal of Computing and Information Science in Engineering, P.18, 2005,

[17] T.S.Mruthyunjaya, "Kinematic Structure of Mechanisms Revisited," Mechanism and Machine Theory, vol. 38, pp, 279-320, 2003
[18] K.Vijayanand "Computer aided structural synthesis of linkages and epicyclic gear transmissions," Ph.D thesis, IISc, Bangalore.

[19] V.P. Agrwal , J.N. Yadav, C.R.Pratap, "Mechanism of Kinematic Chain and Degree of Structural Similarity based on the concept of Link-Link- path Code", Mechanism and Machine Theory, 31(7),pp. 865-871, 1996. 